\title{
Effect of a magnetic field on the magnetostructural phase transition in $\operatorname{Gd}_{5}\left(\operatorname{Si}_{x} \mathbf{G e}_{1-x}\right)_{4}$
}

\author{
Fèlix Casanova, Amílcar Labarta, and Xavier Batlle* \\ Departament de Física Fonamental, Universitat de Barcelona, Diagonal 647, 08028-Barcelona, Catalonia, Spain \\ Jordi Marcos, Lluís Mañosa, and Antoni Planes \\ Departament d' Estructura i Constituents de la Matèria, Universitat de Barcelona, Diagonal 647, 08028-Barcelona, Catalonia, Spain \\ Sophie de Brion \\ Grenoble High Magnetic Field Laboratory, MPI-FKF and CNRS, Boite Postale 166, 38042 Grenoble Cedex 9, France
}

(Received 3 July 2003; revised manuscript received 7 October 2003; published 19 March 2004)

\begin{abstract}
The first-order magnetostructural phase transition in $\mathrm{Gd}_{5}\left(\mathrm{Si}_{x} \mathrm{Ge}_{1-x}\right)_{4}$ alloys with $x \leqslant 0.5$ and the related entropy change $\Delta S$ are analyzed from high-field magnetization curves up to $23 \mathrm{~T}$, and differential scanning calorimetry up to $5 \mathrm{~T}$. The variation of the transition field $H_{t}$ with the transition temperature $T_{t}$ is discussed for fields up to $23 \mathrm{~T}$ for the $x \leqslant 0.5$ range. From these data, the ratio $d H_{t} / d T_{t}$, which is related to the strength of the magnetoelastic coupling, is obtained as a function of $x$. We show that $\Delta S$ obtained from the ClausiusClapyeron equation and the Maxwell relation are equivalent for all compositions $(0 \leqslant x \leqslant 0.5)$, provided the Maxwell relation is evaluated only within the transition region. The $T$ and $H$ dependences of $\Delta S$ are accounted for by modeling the $T$ and $H$ dependences of the magnetization outside the transition.
\end{abstract}

DOI: $10.1103 /$ PhysRevB.69.104416

PACS number(s): 75.30.Sg, 75.30.Kz, 64.70.Kb

\section{INTRODUCTION}

The magnetocaloric effect (MCE) has been studied for decades owing to its potential application to magnetic refrigerants. ${ }^{1}$ The MCE refers both to the isothermal entropy change and to the adiabatic temperature change arising from the application or removal of a magnetic field $H$ on a system with magnetic degrees of freedom. MCE may be maximized in the vicinity of a first-order phase transition, when the transformation is field induced, resulting in a large contribution to the entropy change. ${ }^{1,2}$ Such a giant MCE has been found in $\mathrm{Gd}_{5}\left(\mathrm{Si}_{x} \mathrm{Ge}_{1-x}\right)_{4}$ compounds with $x \leqslant 0.5,{ }^{3-5}$ in MnAs-based materials ${ }^{6,7}$ and in $\mathrm{La}\left(\mathrm{Fe}_{x} \mathrm{Si}_{1-x}\right)_{13}$ alloys. ${ }^{8,9}$

Two different compositional ranges are observed in $\mathrm{Gd}_{5}\left(\mathrm{Si}_{x} \mathrm{Ge}_{1-x}\right)_{4}$ with $x \leqslant 0.5$. For $0.24 \leqslant x \leqslant 0.5$, the firstorder magnetostructural phase transition occurs from a hightemperature paramagnetic (PM) monoclinic phase $\left(P 112_{1} / a\right)$ to a low-temperature ferromagnetic (FM) $\mathrm{Gd}_{5} \mathrm{Si}_{4}$-type orthorhombic-I phase (Pnma), at temperatures ranging linearly from $130 \mathrm{~K} \quad(x=0.24)$ to $276 \mathrm{~K}$ $(x=0.5){ }^{4,10}$ For $x \leqslant 0.2$, the symmetry is $\mathrm{Sm}_{5} \mathrm{Ge}_{4}$-type orthorhombic-II (Pnma), and a second-order PM-toantiferromagnetic (PM-AFM) phase transition occurs at $T_{N}$ (from $\sim 125 \mathrm{~K}$ for $x=0$ to $\sim 135 \mathrm{~K}$ for $x=0.2$ ). Upon further cooling, a first-order structural transition takes place, simultaneously with an AFM-FM transition from the hightemperature orthorhombic-II phase to the low-temperature orthorhombic-I phase. The transition temperature ranges linearly from about $20 \mathrm{~K}(x=0)$ to $120 \mathrm{~K}(x=0.2){ }^{4,5,11}$ The nature of this AFM phase is currently under discussion. ${ }^{11,12}$ In the intermediate range $0.2<x<0.24$, at which the secondorder PM-AFM transition disappears, orthorhombic-II and monoclinic structures coexist. ${ }^{5}$

The structural transition occurs by a shear mechanism ${ }^{13}$ in the whole composition range and it is accompanied by a large volume variation. This fact may be understood consid- ering the layered crystal structure of $\mathrm{Gd}_{5}\left(\mathrm{Si}_{x} \mathrm{Ge}_{1-x}\right)_{4}$ (Ref. 14). In the low-temperature orthorhombic-I phase, which is FM for all compositions, two-dimensional slabs (layers) are connected one to another through $\mathrm{Ge}(\mathrm{Si})-\mathrm{Ge}(\mathrm{Si})$ covalentlike bonds. ${ }^{13}$ For $x \leqslant 0.2$, the interslab bonds are totally broken at the transformation to the orthorhombic-II phase due to the increase in the distance between $\mathrm{Ge}(\mathrm{Si})$ atoms, which stabilizes an AFM phase. However, only half of the $\mathrm{Ge}(\mathrm{Si})-\mathrm{Ge}(\mathrm{Si})$ bonds are broken on the transformation to the monoclinic phase for $0.24 \leqslant x \leqslant 0.5$, leading to a PM phase. ${ }^{14}$ The fieldinduced nature of these magnetostructural transitions results in strong magnetostriction ${ }^{10,11}$ and giant magnetoresistance, ${ }^{15-17}$ besides the giant MCE. The MCE is associated with the large contribution of the entropy change at the first-order transition, $\Delta S$, whose determination has been a controversial issue., $318-21$ Recently, we showed that in $\mathrm{Gd}_{5}\left(\mathrm{Si}_{0.45} \mathrm{Ge}_{0.55}\right)_{4}$ (Ref. 22), the values of $\Delta S$ calculated through the Clausius-Clapeyron equation matched the calorimetric measurements of $\Delta S$, while the Maxwell relation also accounted for the entropy change outside the transition region, due to the field and temperature dependence of the magnetization. Moreover, for all compositions with $x \leqslant 0.5$, a scaling of $\Delta S$ was achieved by tuning the transition temperature $T_{t}$ through $x$ and $H$, which suggested that magnetovolume effects and substitution-related effects are equivalent. ${ }^{23}$

In this work, we study the effect of the magnetic field on the magnetostructural transition in $\mathrm{Gd}_{5}\left(\mathrm{Si}_{x} \mathrm{Ge}_{1-x}\right)_{4}$ alloys with $x \leqslant 0.5$. In particular, the variation of the magnetic field at which the transition takes place, $H_{t}$, with $T_{t}$ is discussed as a function of $x$. In these compounds, the value of $\Delta S$ measured when the transition is field induced coincides with the value measured when it is induced by the application of pressure ${ }^{10}$ Therefore, through the Clausius-Clapeyron equation, it is shown that $d H_{t} / d T_{t}$ is related to the strength of the magnetoelastic coupling. ${ }^{23}$ We also compare the values of 
$\Delta S$ obtained from calorimetric measurements, from the Clausius-Clapeyron equation and from the Maxwell relation, for all compositions with $x \leqslant 0.5$. Finally, we present a generalization of a previous phenomenological model (Ref. 22) which enables us to assess the conditions in which the Clausius-Clapeyron and Maxwell approaches will give coincident results for $\Delta S$.

\section{EXPERIMENTAL DETAILS}

$\mathrm{Gd}_{5}\left(\mathrm{Si}_{x} \mathrm{Ge}_{1-x}\right)_{4}$ samples $(x=0,0.05,0.1,0.18,0.2,0.25$, $0.3,0.365$, and 0.45 ) were synthesized by arc melting the pure elements in the desired stoichiometry under a highpurity argon atmosphere. The samples were placed in a water-cooled copper crucible and each one was melted several times to ensure a good homogeneity. The weight losses after arc-melting were negligible. As-prepared buttons were cut into slices and some of them were heat treated for up to $9 \mathrm{~h}$ at $950^{\circ} \mathrm{C}$ under $10^{-5}$ mbar, inside a quartz tube in an electrical resistance furnace. After annealing, the quartz tube was quickly taken out of the furnace to room temperature. The crystallographic structure of the samples was studied by room-temperature $\mathrm{x}$-ray diffraction (XRD). The samples with $x \leqslant 0.2$ displayed the expected room-temperature orthorhombic-II structure $\left(\mathrm{Sm}_{5} \mathrm{Ge}_{4}\right.$-type Pnma $)$, in agreement with Refs. 5 and 11 . The rest of the samples $(0.24 \leqslant x$ $\leqslant 0.5)$ displayed the monoclinic structure $\left(P 112_{1} / a\right){ }^{4,10}$ Minoritary $\mathrm{Gd}_{5}(\mathrm{Si}, \mathrm{Ge})_{3}$ and $\mathrm{Gd}_{1}(\mathrm{Si}, \mathrm{Ge})_{1}$ phases (less than $10 \mathrm{wt} . \%)$ are also detected by XRD and electron-beam microprobe analysis. The magnetization measurements were performed at the Grenoble High Magnetic Field Laboratory. $M(H)$ curves were recorded from 4.2 to $310 \mathrm{~K}$ with a temperature step of 3-5 K up to $23 \mathrm{~T}$, both under increasing and decreasing $H$. Calorimetric measurements were performed using a high-sensitivity differential scanning calorimeter (DSC) specifically designed to study solid-solid phase transitions. ${ }^{24}$ Heat flow $\dot{Q}(t)$ and temperature $T(t)$ were acquired at $0.33 \mathrm{~Hz}$. Heating and cooling runs were performed within $4.2-300 \mathrm{~K}$ under fields up to $5 \mathrm{~T}$ in a LHe cryostat. Neither the thermometry nor the heat flow sensors were affected by $H$.

\section{RESULTS AND DISCUSSION}

\section{A. Magnetoelastic coupling}

DSC data under different magnetic fields $(0-5 \mathrm{~T})$ were recorded for all samples. In these measurements, first-order transitions give rise to a large peak in thermal curves $(d Q / d T) .{ }^{25}$ Second-order transitions are observed as small $\lambda$-type jumps in the $d Q / d T$ base line. The shape of the thermal curves for all compositions with $x \leqslant 0.2$ reveals the firstorder nature of the low-temperature AFM-FM transition and the second-order nature of the high-temperature PM-AFM transition (see Fig. 1 for $x=0,0.05$, and 0.2 , where the second-order transitions are also labeled). For the rest of compositions $(0.24 \leqslant x \leqslant 0.5)$, only one peak is displayed, corresponding to the first-order PM-FM transition. For all samples, a hysteresis of 2-4 $\mathrm{K}$ between cooling and heating runs is observed. We note that for $x=0$, the cooling process

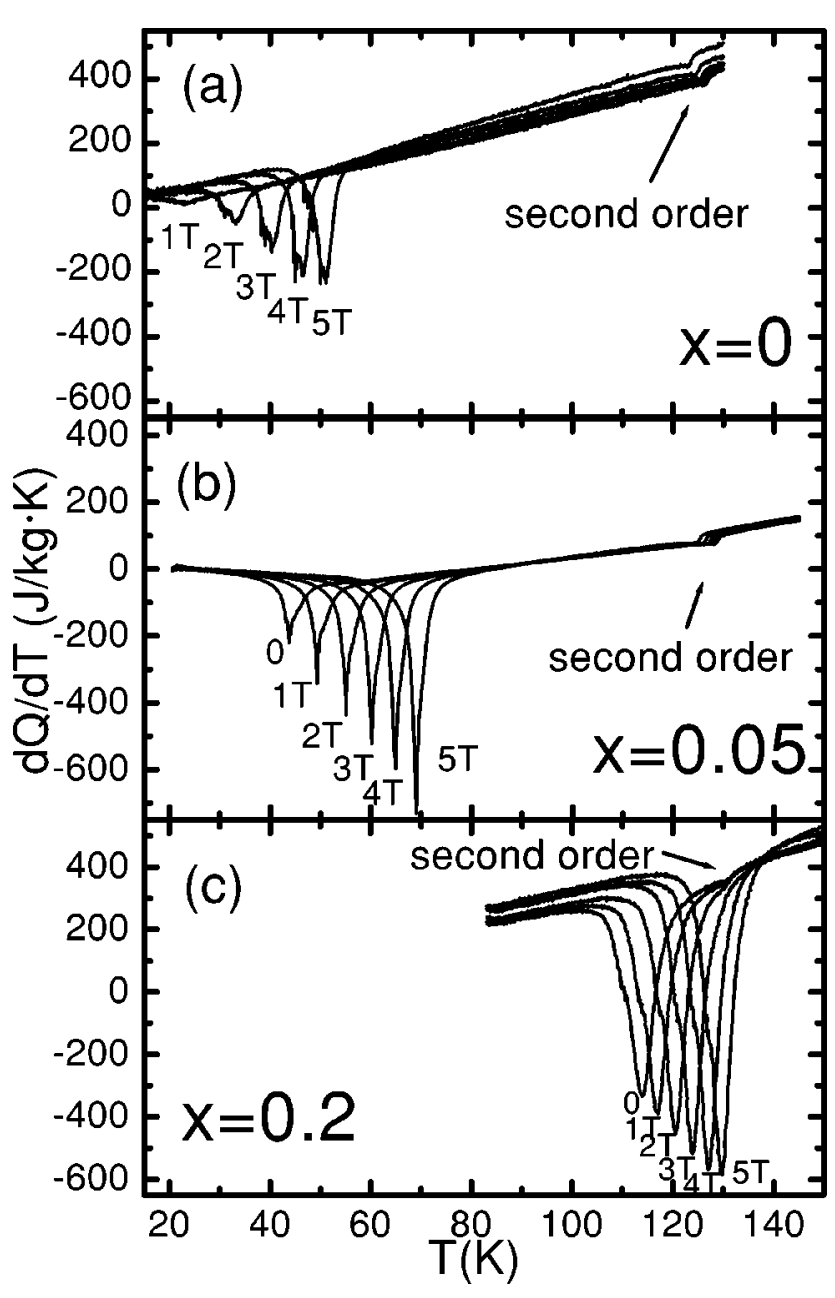

FIG. 1. DSC data on cooling at selected applied fields up to $5 \mathrm{~T}$ for $\mathrm{Gd}_{5}\left(\mathrm{Si}_{x} \mathrm{Ge}_{1-x}\right)_{4}$ : (a) $x=0$, (b) $x=0.05$, and (c) $x=0.2$. The second-order transition is labeled for each composition.

at zero field does not show any first-order peak [hence it is not displayed in Fig. 1(a)]. This is due to the fact that the FM ground state for $x=0$ cannot be achieved by cooling to low temperature at zero field, since the sample remains AFM. The application of a field of $\sim 1 \mathrm{~T}$ is needed in order to stabilize the FM phase through an irreversible transition. ${ }^{26,27}$ Figure 1 shows how the first-order transition comes progressively closer to the second-order transition as Si content $x$ is increased. In particular, Fig. 1(c) shows the overlap of the first-order peak and the second-order jump when a field of $\sim 3 \mathrm{~T}$ (or larger) is applied for $x=0.2$.

$M(H)$ isotherms for all compositions (see, for example, Fig. 2 for $x=0.1$ and 0.3) exhibit a change in the magnetization, $\Delta M,^{3,18,22,28,29}$ which spreads over a field range, $\Delta H_{t}$, around the magnetostructural transition. The hysteresis between $M(H)$ isotherms obtained by increasing and decreasing $H$ evidences the first-order nature of the transition. For $x \leqslant 0.2$ [for example $x=0.1$ in Fig. 2(a)], the first-order field-induced transition occurs between AFM and FM phases, while for $0.24 \leqslant x \leqslant 0.5$ [see Fig. 2(b) for $x=0.3$ ] it occurs from PM to FM phases. This fact affects the behavior of $\Delta M$ at the transition, the variation of magnetization being more abrupt for the AFM-FM case. In particular, in Fig. 2(a) 


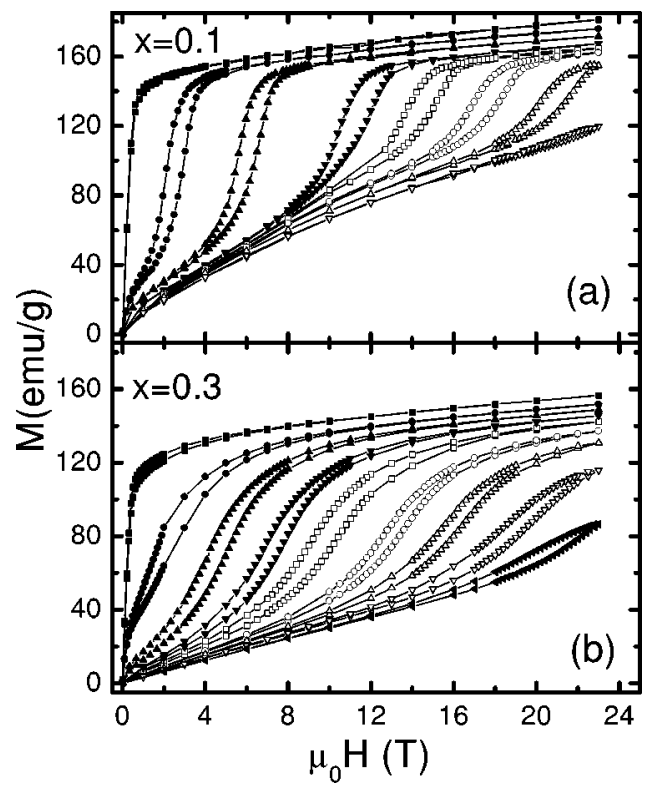

FIG. 2. (a) Magnetization isotherms for $\mathrm{Gd}_{5}\left(\mathrm{Si}_{0.1} \mathrm{Ge}_{0.9}\right)_{4}$ under increasing and decreasing field for selected temperatures: $148.5 \mathrm{~K}$, 162.7 K, 173.1 K, 181.9 K, 190.8 K, 199.9 K, 208.8 K, 218.3 K, and $228.8 \mathrm{~K}$, from top to bottom. (b) Magnetization isotherms for $\mathrm{Gd}_{5}\left(\mathrm{Si}_{0.3} \mathrm{Ge}_{0.7}\right)_{4}$ under increasing and decreasing field for selected temperatures: $70.8 \mathrm{~K}, 84.3 \mathrm{~K}, 97.0 \mathrm{~K}, 109.8 \mathrm{~K}, 115.7 \mathrm{~K}, 121.0 \mathrm{~K}$, 127.2 K, and $133.0 \mathrm{~K}$. The first-order field-induced nature of the transition is evident.

for $x=0.1$, the shape of the magnetization curves at the transition becomes smoother as the nature of the transition changes from AFM-FM (low- $T$ isotherms) to PM-FM (high$T$ isotherms).

From both sets of measurements [DSC and $M(H)]$, the dependence of $T_{t}$ on the transition field $H_{t}$ can be evaluated independently. From magnetization isotherms, $H_{t}(T)$ is defined at each temperature as the field corresponding to the inflection point within the transition region. Due to the hysteresis between increasing and decreasing field, two different values of $H_{t}$ are obtained. From DSC, $T_{t}(H)$ is estimated at each applied field as the peak position in $d Q / d T$ curves. Due to the thermal hysteresis, two different values of $T_{t}$ are obtained. Figure 3(a) displays the transition field as a function of the transition temperature obtained from both DSC and $M(H)$ curves. Notice the good agreement between isofield and isothermal data. Interestingly, for $0.24 \leqslant x \leqslant 0.5$, where only the PM-to-FM transition occurs, $H_{t}\left(T_{t}\right)$ shows a linear behavior over the whole field range, while for $x \leqslant 0.2$, the slope of $H_{t}\left(T_{t}\right)$ varies progressively from a low-field value (AFM-FM transition) to a high-field value (PM-FM transition). This effect is illustrated in Fig. 3(b), which shows a detail of Fig. 3(a) for $x=0.1$ and $x=0.18$ curves. Such a progressive change in the slope is due to the fact that, at high fields, the magnetostructural transition overlaps the secondorder PM-AFM transition ${ }^{23}$ [Fig. 1(c)], giving rise to a unique PM-FM transition.

Figure 3(c) compiles, for all compositions, the values of the slope, $d H_{t} / d T_{t}$, as a function of $x$, determined from the data in Fig. 3(a). For $x \leqslant 0.2$, two limiting values of $d H_{t} / d T_{t}$
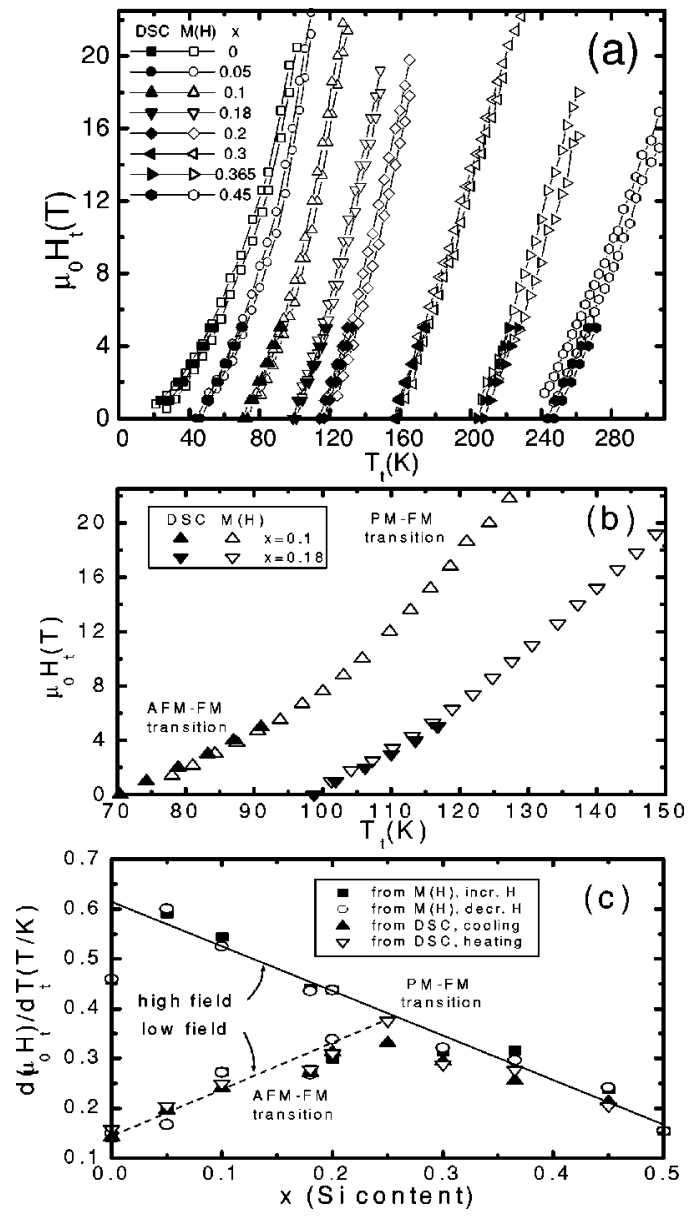

FIG. 3. (a) Transition field $H_{t}$ as a function of the transition temperature $T_{t}$ for $\mathrm{Gd}_{5}\left(\mathrm{Si}_{x} \mathrm{Ge}_{1-x}\right)_{4}$ (from $x=0$ to $x=0.45$ ) obtained from magnetization isotherms (increasing and decreasing $H$ ) and DSC isofield data (cooling and heating). (b) Detail of panel (a) showing $H_{t}\left(T_{t}\right)$ for $x=0.1$ and $x=0.18$, on increasing $H$ and cooling. (c) Slope of $H_{t}\left(T_{t}\right)$ calculated from the previous data. For $x$ $=0.25,0.3,0.365,0.45$, and 0.5 (the latter from Ref. 18) a single slope is obtained, which corresponds to the PM-FM transition. For $x=0,0.05,0.1,0.18$, and 0.2 two limiting slopes are obtained: a low-field value (associated with the AFM-FM transition) and a high-field value (associated with the PM-FM transition). Solid and dotted lines are a guide to the eye.

corresponding to the low- and high-field regimes are displayed, while a single value of $d H_{t} / d T_{t}$ is found for 0.24 $\leqslant x \leqslant 0.5$. Datum for $x=0.5$ is taken from Ref. 18 . We note the linear dependence of $d H_{t} / d T_{t}$ on $x$, which is decreasing for the PM-FM transition [solid line in Fig. 3(c)], while it is increasing for the AFM-FM transition [dashed line in Fig. $3(\mathrm{c})$. Both lines meet at the composition range where the second-order transition disappears $(0.2<x<0.24)$, in agreement with the phase diagram. ${ }^{11}$ The value of $d H_{t} / d T_{t}$ for $x=0$ at high fields is lower than expected because a field higher than $23 \mathrm{~T}$ (the maximum available in the present work) must be applied to fully induce the PM-FM transition.

The strength of the magnetoelastic coupling is associated with the field dependence of $T_{t}$ (i.e., a strong magnetoelastic coupling yields a small value of $d H_{t} / d T_{t}$ ). Consequently, the decrease in $d H_{t} / d T_{t}$ with increasing $x$ for the PM-FM 
transition indicates a strengthening of the magnetoelastic coupling. This may be explained by considering that FM exchange interactions are stronger for increasing $x$, as suggested by the magnetic phase diagram, where $T_{t}$ increases linearly with $x .{ }^{11}$ The fact that $d H_{t} / d T_{t}$ for the PM-FM transition has continuous behavior, although the PM phase is monoclinic for $x \leqslant 0.2$ and orthorhombic-II for $0.24 \leqslant x$ $\leqslant 0.5$, suggests that the magnetoelastic coupling is weakly dependent on the actual crystallographic structure. Concerning the AFM-FM transition, and taking into account that the structural transition is very similar to that occurring in the PM-FM case, the increase in $d H_{t} / d T_{t}$ with $x$ may be related to the fact that the transition involves two ordered magnetic phases (FM and AFM).

\section{B. Entropy change}

Let us now analyze $\Delta S$ involved in the first-order transition. After a proper correction of the base line, $\Delta S$ is obtained from DSC data as

$$
\Delta S=\int_{T_{i}}^{T_{f}} \frac{1}{T} \frac{d Q}{d T} d T
$$

where $T_{i}$ and $T_{f}$ are temperatures located above (below) and below (above) the DSC peak measured on cooling (heating). $\Delta S$ as a function of $T_{t}$ is displayed for $x=0.05$ and $x=0$ in Fig. 4 (open triangles). The results for the rest of compositions were published elsewhere. ${ }^{23}$

The entropy change as a function of $T$ for each $x$ may be also obtained from magnetization data. We note that $\Delta S$ at the first-order magnetostructural transition and the total entropy change due to the variation of the magnetization by applying a magnetic field, $\Delta S\left(0 \rightarrow H_{\max }\right)$, may be different. ${ }^{22}$

On one hand, $\Delta S$ can be obtained by using the ClausiusClapeyron equation:

$$
\Delta S=-\Delta M \frac{d H_{t}}{d T_{t}}
$$

the results (open squares in Fig. 4) being in good agreement with the calorimetric measurements within the experimental error.

On the other hand, $\Delta S\left(0 \rightarrow H_{\max }\right)$ corresponding to a magnetization process from zero field up to $H_{\max }$ can be evaluated by using the Maxwell relation:

$$
\Delta S\left(0 \rightarrow H_{\max }\right)=\int_{0}^{H_{\max }}\left(\frac{\partial M}{\partial T}\right)_{H} d H .
$$

The results of Eq. (3) (dashed lines in Fig. 4), which depend on the maximum applied field $\left(H_{\max }\right)$, are clearly above $\Delta S$ when $H_{\max }$ is high enough as to fully induce the magnetostructural transition. The extra contribution to the entropy change with respect to $\Delta S$ is due to the $T$ and $H$ variation of the magnetization in the two phases outside the transition region. ${ }^{22}$ However, when the Maxwell relation is evaluated only within $\Delta H_{t}$ centered at the transition field, ${ }^{30}$ the results (thick solid lines in Fig. 4) agree with those obtained from

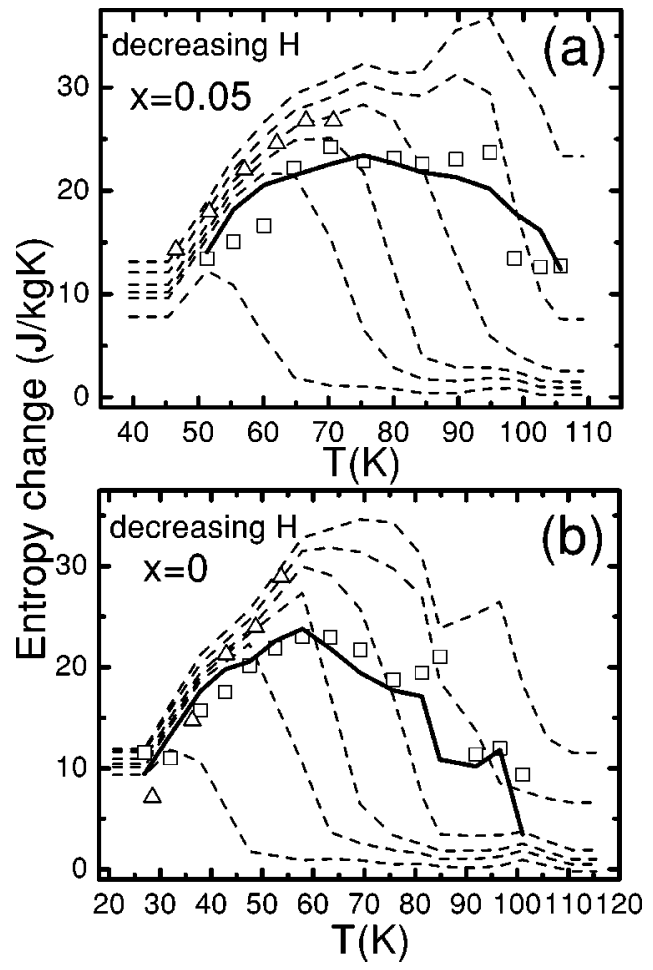

FIG. 4. Entropy change in $\mathrm{Gd}_{5}\left(\mathrm{Si}_{x} \mathrm{Ge}_{1-x}\right)_{4}$ for (a) $x=0.05$ and (b) $x=0$ calculated by using DSC measurements under field (open triangles); the Clausius-Clapeyron equation (open squares); the Maxwell relation integrating from $H_{\max }(20,15,10,7,5$, and $2 \mathrm{~T}$, from right to left, respectively) to zero (dashed lines); and the Maxwell relation integrating only within the transition region (solid line).

both the Clausius-Clapeyron equation and DSC. This finding proves the equivalence of the Maxwell relation evaluated within the transition region and the Clausius-Clapeyron equation, not only in the $0.24 \leqslant x \leqslant 0.5$ region (as already suggested in Ref. 22 for $x=0.45$ ), but also in the $x \leqslant 0.2$ region, where other magnetic phases are involved in the transition.

We note that $\Delta S\left(0 \rightarrow H_{\max }\right)$ obtained from the Maxwell relation for $x=0$ and $x=0.05$ shows a double-peak structure, when integrating from zero to very high fields (15 and $20 \mathrm{~T}$ ). This fact may evidence two magnetic transitions in the system. ${ }^{31}$ The peak at high temperature is associated with the first-order transition and it shifts to higher temperatures as the maximum applied field $\left(H_{\max }\right)$ increases. The lowtemperature peak is related to the field-induced transition from the AFM phase to a phase with competing short-range AFM and FM correlations, which appears at very high fields. A detailed study of this transition will be reported elsewhere. ${ }^{31}$ This effect is also evident in $\Delta S$ determined from the Clausius-Clapeyron equation at high $T_{t}$, i.e., very high $H_{t}$.

\section{Model}

In order to account for the main features of the entropy change reported in Sec. III B, we propose a phenomenological model that considers the overall behavior of the magne- 


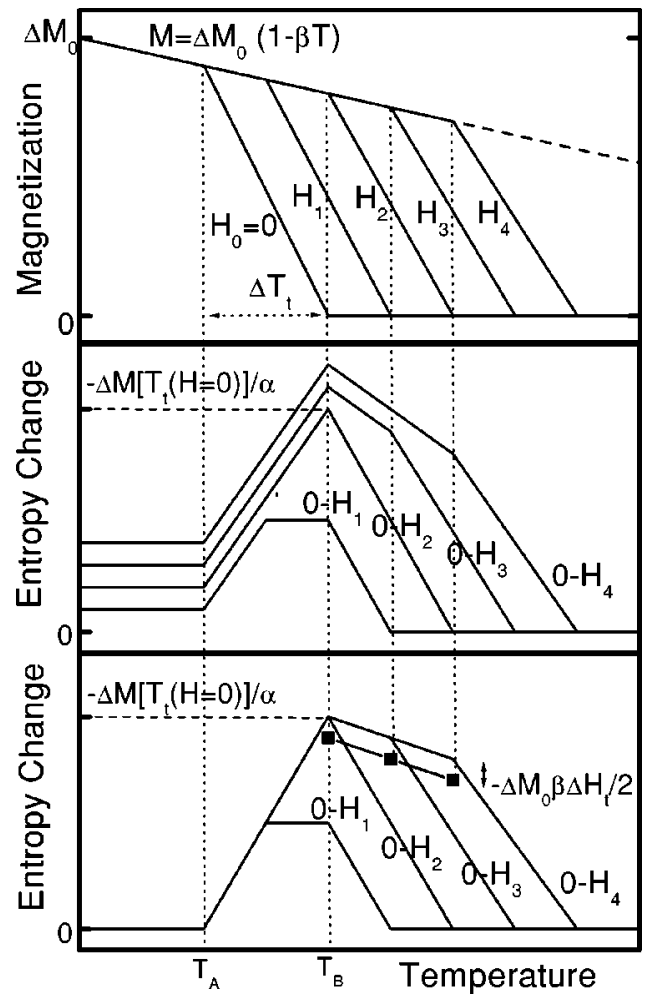

FIG. 5. Upper panel shows the modeled temperature dependence of the magnetization across the transition region at different fields, as described in the text. Middle panel shows the corresponding entropy change $\Delta S\left(0 \rightarrow H_{\max }\right)$ calculated from the Maxwell relation [Eq. (3)]. Lower panel: Solid lines stand for the entropy change obtained by integrating the Maxwell relation excluding the contribution to $\Delta S$ evaluated outside the transition region. Connected squares stand for $\Delta S$ obtained from the Clausius-Clapeyron equation [Eq. (2)]. The difference between these values is indicated in the figure.

tization in a system with a first-order field-induced transition. This model is a generalization of that given in Ref. 22. The present model includes both the $T$ and $H$ dependences of the magnetization outside the transition region and the decrease of $\Delta M$ with $T$. The upper panel in Fig. 5 shows the modeled $M(T)$ curves at different $H$. The transition temperature is assumed to shift linearly with the transition field, $d T_{t} / d H_{t}$ $\equiv \alpha=$ constant. The magnetization of the low-temperature phase is assumed to decrease linearly with $T$ as $M(T)$ $=\Delta M_{0}(1-\beta T)$, being zero at the high-temperature phase. The transition between both phases extends within a temperature range $\Delta T_{t}=\alpha \Delta H_{t}$, which is assumed to be constant according to the experimental results. In this model, $T_{t}(H)$ is defined for each curve as the temperature at the center of the transition region. As $\alpha$ is considered to be constant, the model should account for the behavior of the entropy change for $0.24 \leqslant x \leqslant 0.5$ (see Fig. 3).

The results of the model are compiled in the middle panel in Fig. 5. The behavior, which depends on the temperature range and the maximum applied field, can be summarized as follows.

(i) For temperatures at which the system is in the lowtemperature phase $\left[T \leqslant T_{A}\right.$, with $\left.T_{A} \equiv T_{t}(H=0)-\Delta T_{t} / 2\right]$,
$\Delta S$ is independent of $T$ and increases linearly with the maximum applied field as

$$
\Delta S\left(0 \rightarrow H_{\max }\right)=-\Delta M_{0} \beta H_{\max } .
$$

(ii) In the range $T_{A} \leqslant T \leqslant T_{B}\left[T_{B} \equiv T_{t}(H=0)+\Delta T_{t} / 2\right]$, which is the temperature spread of the transition at zero field (see upper panel in Fig. 5), the entropy change increases linearly up to $T_{t}\left(H_{\max }\right)-\Delta T_{t} / 2$ and reaches a plateau, with a value increasing with $H_{\max }$ (see $H_{1}$ in Fig. 5). The limiting case of this behavior is obtained when the maximum applied field is strong enough to induce the whole transition (isothermally). Then $T_{t}\left(H_{\max }\right)-\Delta T_{t} / 2=T_{B}$ and the value is

$$
\begin{aligned}
\Delta S\left(0 \rightarrow H_{\max }\right) & =-\frac{\Delta M_{0}\left[1-\beta T_{t}(H=0)\right]}{\alpha} \\
& \equiv-\frac{\Delta M\left[T_{t}(H=0)\right]}{\alpha}
\end{aligned}
$$

(see case for $\mathrm{H}_{2}$ in Fig. 5). For higher fields, the transition is also completed at $T_{B}$ and there is an additional contribution to $\Delta S$ due to the field and temperature dependences of $M$ of the low-temperature phase (see cases for $\mathrm{H}_{3}$ and $\mathrm{H}_{4}$ in Fig. 5). Therefore

$$
\Delta S\left(0 \rightarrow H_{\max }\right)=-\frac{\Delta M\left[T_{t}(H=0)\right]}{\alpha}-\Delta M_{0} \beta\left(H_{\max }-\Delta H_{t}\right) .
$$

(iii) For temperatures at which the system is in the high- $T$ phase at zero field $\left(T \geqslant T_{B}\right)$ and for low fields (see $H_{1}$ and $\mathrm{H}_{2}$ in Fig. 5), $\Delta S$ decreases linearly to zero with increasing $T$, vanishing at $T_{t}\left(H_{\max }\right)+\Delta T_{t} / 2$, which corresponds to the minimum temperature at which $H_{\max }$ is not enough to induce the transition. For fields where the transition is complete (see $H_{3}$ and $H_{4}$ in Fig. 5), $\Delta S$ shows plateaulike behavior with a slope $2 \Delta M_{0} \beta / \alpha$ up to $T_{t}\left(H_{\max }\right)-\Delta T_{t} / 2$. Above this temperature, the field is not enough to complete the transition and $\Delta S$ decreases linearly to zero, vanishing at $T_{t}\left(H_{\max }\right)$ $+\Delta T_{t} / 2$.

The lower panel in Fig. 5 shows the entropy change (solid lines) calculated by integrating the Maxwell relation excluding the contribution to $\Delta S$ evaluated outside the transition region. The values of $\Delta S$ calculated by using the ClausiusClapeyron equation [Eq. (3)] are also plotted as connected squares. Three main features are to be noted: (i) for temperatures at which the transition does not occur $\left(T \leqslant T_{A}\right), \Delta S(0$ $\left.\rightarrow H_{\max }\right)=0$; (ii) for temperatures at which the transition can be completely field induced $\left(T \geqslant T_{B}\right)$, and for $H_{\max }$ strong enough to complete it, the plateaulike regions of all curves overlap, yielding a slope $\Delta M_{0} \beta / \alpha$; and (iii) $\Delta S$ values obtained from the Clausius-Clapeyron equation decrease with the same slope, but lowered by $\delta=\Delta M_{0} \beta \Delta H_{t} / 2$. The model accounts for the behavior of the experimental results shown in Fig. 6, and Fig. 2 in Ref. 22 for $0.24 \leqslant x \leqslant 0.5$. We note that in Fig. 6 the values of $\Delta S$ calculated from the ClausiusClapeyron equation at low $T$ increase with $T$ due to the fact that, just above the zero-field transition temperature, a fraction of the sample has not yet been transformed to the PM 


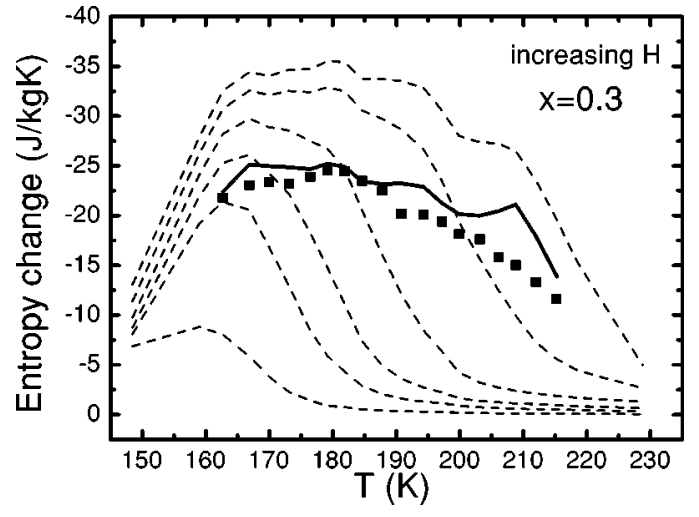

FIG. 6. Entropy change for $\mathrm{Gd}_{5}\left(\mathrm{Si}_{0.3} \mathrm{Ge}_{0.7}\right)_{4}$, calculated by using the Clausius-Clapeyron equation (solid squares); the Maxwell relation integrating from zero to $H_{\max }(20,15,10,7,5$, and $2 \mathrm{~T}$, dashed lines from right to left, respectively); and the Maxwell relation integrating only within the transition region (solid line).

phase and still remains $\mathrm{FM}^{29}$ We also note that for $x \leqslant 0.2$, although $\alpha$ is not constant, the model accounts for the main features of the PM-FM transition. An extension of the present model should consider $\alpha$ as a function of $H_{t}$ and $T_{t}$.

In order to improve the model, a linear $H$ dependence of the low- $T$ magnetization outside the transition region is introduced as $M(T, H)=\Delta M_{0}(1-\beta T+\gamma H)$. This is a more realistic assumption for the magnetization curves (Fig. 2). However, the overall behavior remains unchanged. In this case, Eq. (5) turns into

$$
\Delta S\left(0 \rightarrow H_{\max }\right)=-\frac{\Delta M\left[T_{t}(H=0)\right]}{\alpha}-\frac{\Delta M_{0} \gamma \Delta H_{t}}{2 \alpha},
$$

the slope of the plateaulike region of $\Delta S$ values evaluated within the transition region from Eq. (3) is now $\Delta M_{0} \beta^{\prime} / \alpha$, with $\beta^{\prime}=\beta-\gamma / \alpha$, and $\delta^{\prime}=\Delta M_{0} \beta^{\prime} \Delta H_{t} / 2$. This shift $\delta^{\prime}$ is due to the fact that $H$ heightens $T_{t}$, resulting in a reduction of $\Delta M$. Since $\Delta T_{t}$ is assumed to be constant, $\Delta M / \Delta T_{t}$ in the transition region decreases correspondingly. This $H$ dependence remains included within the transition region (as $\partial M / \partial T)$ and still gives an extra term to the entropy change when integrated in Eq. (3), but does not contribute to the Clausius-Clapeyron equation [Eq. (2)]. Nevertheless, $\delta^{\prime}$ is small for $\mathrm{Gd}_{5}\left(\mathrm{Si}_{x} \mathrm{Ge}_{1-x}\right)_{4}$ alloys. For example, for $x=0.45$ (see Fig. 2 in Ref. 22), $\Delta M_{0} \beta^{\prime}=0.753 \mathrm{emu} / \mathrm{g} \mathrm{K}$ and $\Delta H_{t}$ $\sim 4 \mathrm{~T}$, yielding $\delta^{\prime} \sim 1.5 \mathrm{~J} / \mathrm{kg} \mathrm{K}$, which is within the experimental error of the entropy change. For $x=0.3, \Delta M_{0} \beta^{\prime}$ $=1.163 \mathrm{emu} / \mathrm{g} \mathrm{K}$ and $\Delta H_{t} \sim 7 \mathrm{~T}$, resulting in $\delta^{\prime}$ $\sim 4.1 \mathrm{~J} / \mathrm{kg} \mathrm{K}$, which may account for the slight difference observed in Fig. 6. Generally, $\delta^{\prime}$ is expected to be small, since it is proportional to the variation of the magnetization outside the transition region and this variation is small in a FM phase. This may be extended to other field-induced transitions that involve a FM phase.

\section{CONCLUSIONS}

The variation of the transition field with the transition temperature, $d H_{t} / d T_{t}$, has been studied in $\mathrm{Gd}_{5}\left(\mathrm{Si}_{x} \mathrm{Ge}_{1-x}\right)_{4}$ for all the range of compositions where the first-order transition occurs, i.e., $0 \leqslant x \leqslant 0.5$. Taking into account the behavior of $d H_{t} / d T_{t}$ as a function of $x$ and that $\Delta M$ decreases monotonously with $T_{t}$, it is shown that $d H_{t} / d T_{t}$ governs the scaling of $\Delta S$ with $T_{t}$ reported in Ref. 23, giving further evidence that the origin of this scaling is the magnetoelastic nature of the transition. Moreover, two distinct behaviors for $d H_{t} / d T_{t}$ have been found on the two compositional ranges where the magnetostructural transition occurs, thus showing the difference in the strength of the magnetoelastic coupling of this system. The equivalence of the Clausius-Clapyeron equation and the Maxwell relation evaluated only within the transition region has shown to be valid in $\mathrm{Gd}_{5}\left(\mathrm{Si}_{x} \mathrm{Ge}_{1-x}\right)_{4}$ for both $0 \leqslant x \leqslant 0.2$ and $0.24 \leqslant x \leqslant 0.5$. A generalization of a previous model which includes the $T$ and $H$ dependences of the magnetization outside the transition accounts for the $T$ and $H$ dependences of the entropy change.

\section{ACKNOWLEDGMENTS}

The financial support of the Spanish CICYT (Grant Nos. MAT2000-0858 and MAT2001-3251) and Catalan DURSI (Grant No. 2001SGR00066) is recognized. The Grenoble High Magnetic Field Laboratory, through the Improving Human Potential Program of the European Community, is acknowledged. F.C. and J.M. acknowledge DURSI for financial support.
*Electronic address: xavier@ ffn.ub.es

${ }^{1}$ V.K. Pecharsky and K.A. Gschneidner, Jr., J. Magn. Magn. Mater. 200, 44 (1999).

${ }^{2}$ A.M. Tishin, Handbook of Magnetic Materials, edited by K.H.J. Buschow (North Holland, Amsterdam, 1999), Vol. 12, pp. 395524.

${ }^{3}$ V.K. Pecharsky and K.A. Gschneidner, Jr., Phys. Rev. Lett. 78, 4494 (1997).

${ }^{4}$ V.K. Pecharsky and K.A. Gschneidner, Jr., Appl. Phys. Lett. 70, 3299 (1997).

${ }^{5}$ V.K. Pecharsky and K.A. Gschneidner, Jr., J. Alloys Compd. 260, 98 (1997)

${ }^{6}$ H. Wada and Y. Tanabe, Appl. Phys. Lett. 79, 3302 (2001).

${ }^{7}$ O. Tegus, E. Brück, K.H.J. Buschow, and F.R. de Boer, Nature
(London) 415, 450 (2002).

${ }^{8}$ F.X. Hu, B.G. Shen, J.R. Sun, Z.H. Cheng, G.H. Rao, and X.X. Zhang, Appl. Phys. Lett. 78, 3675 (2001).

${ }^{9}$ S. Fujieda, A. Fujita, and K. Fukamichi, Appl. Phys. Lett. 81, 1276 (2002).

${ }^{10}$ L. Morellon, P.A. Algarabel, M.R. Ibarra, J. Blasco, B. GarcíaLanda, Z. Arnold, and F. Albertini, Phys. Rev. B 58, R14 721 (1998).

${ }^{11}$ L. Morellon, J. Blasco, P.A. Algarabel, and M.R. Ibarra, Phys. Rev. B 62, 1022 (2000).

${ }^{12}$ The magnetic structure might correspond to that of either a canted ferrimagnet, as proposed for $\mathrm{Nd}_{5} \mathrm{Ge}_{4}$ in P. SchobingerPapamantellos and A. Niggli, J. Phys. Chem. Solids 42, 583 (1981) or a canted antiferromagnet, as proposed for the Ge-rich 
region of the $\mathrm{Tb}_{5}\left(\mathrm{Si}_{x} \mathrm{Ge}_{1-x}\right)_{4}$ in P. Schobinger-Papamantellos, ibid. 39, 197 (1978) and C. Ritter, L. Morellon, P.A. Algarabel, C. Magen, and M.R. Ibarra, Phys. Rev. B 65, 094405 (2002).

${ }^{13}$ W. Choe, V.K. Pecharsky, A.O. Pecharsky, K.A. Gschneidner, Jr., V.G. Young, Jr., and G.J. Miller, Phys. Rev. Lett. 84, 4617 (2000)

${ }^{14}$ V.K. Pecharsky and K.A. Gschneidner, Jr., Adv. Mater. (Weinheim, Ger.) 13, 683 (2001).

${ }^{15}$ L. Morellon, J. Stankiewicz, B. García-Landa, P.A. Algarabel, and M.R. Ibarra, Appl. Phys. Lett. 73, 3462 (1998).

${ }^{16}$ E.M. Levin, V.K. Pecharsky, and K.A. Gschneidner, Jr., Phys. Rev. B 60, 7993 (1999).

${ }^{17}$ L. Morellon, P.A. Algarabel, C. Magen, and M.R. Ibarra, J. Magn. Magn. Mater. 237, 181 (2001).

${ }^{18}$ A. Giguère, M. Földeàki, B. Ravi Gopal, R. Chahine, T.K. Bose, A. Frydman, and J.A. Barclay, Phys. Rev. Lett. 83, 2262 (1999).

${ }^{19}$ M. Földeàki, R. Chahine, T.K. Bose, and J.A. Barclay, Phys. Rev. Lett. 85, 4192 (2000).

${ }^{20}$ K.A. Gschneidner, Jr., V.K. Pecharsky, E. Brück, H.G.M. Duijn, and E.M. Levin, Phys. Rev. Lett. 85, 4190 (2000).

${ }^{21}$ J.R. Sun, F.X. Hu, and B.G. Shen, Phys. Rev. Lett. 85, 4191 (2000).
${ }^{22}$ F. Casanova, X. Batlle, A. Labarta, J. Marcos, Ll. Mañosa, and A. Planes, Phys. Rev. B 66, 100401(R) (2002).

${ }^{23}$ F. Casanova, X. Batlle, A. Labarta, J. Marcos, L1. Mañosa, and A. Planes, Phys. Rev. B 66, 212402 (2002).

${ }^{24}$ J. Marcos, F. Casanova, X. Batlle, A. Labarta, A. Planes, and L1. Mañosa, Rev. Sci. Instrum. 74, 4768 (2003).

${ }^{25}$ From the recorded $\dot{Q}(t)$ and $T(t)$, the heating/cooling rate $d T / d t$ and $d Q / d T=\dot{Q}(d T / d t)^{-1}$ are numerically computed.

${ }^{26}$ E.M. Levin, V.K. Pecharsky, K.A. Gschneidner, Jr., and G.J. Miller, Phys. Rev. B 64, 235103 (2001).

${ }^{27}$ E.M. Levin, K.A. Gschneidner, Jr., and V.K. Pecharsky, Phys. Rev. B 65, 214427 (2002).

${ }^{28}$ V.K. Pecharsky and K.A. Gschneidner, Jr., Adv. Cryog. Eng. 43, 1729 (1998).

${ }^{29}$ E.M. Levin, V.K. Pecharsky, and K.A. Gschneidner, Jr., Phys. Rev. B 62, R14 625 (2000).

${ }^{30}$ For each magnetization curve, the field range $\Delta H_{t}$ is evaluated as the difference between the two fields limiting the transition region, which correspond to maximum values of the curvature.

${ }^{31}$ F. Casanova, A. Labarta, X. Batlle, J. Marcos, L1. Mañosa, A. Planes, and S. de Brion (unpublished). 